\title{
Advances in Fibre Based Pulse Shaping Technology and its Applications in Optical Communications
}

\author{
D.J. Richardson, P. Petropoulos, F. Parmigiani, P.J. Almeida, C. Tian, T.T. Ng, Z. Zhang and M. Ibsen \\ Optoelectronics Research Centre, University of Southampton, Highfield, Southampton, SO17 1BJ, UK \\ Tel: +44 2380 5954524, Fax: +44 2380 59342,e-mail:djr@orc.soton.ac.uk
}

\begin{abstract}
Fibre grating technology can be used to design and fabricate reflective filters with accurately controlled complex phase and amplitude responses and thereby provide a powerful means to reshape short optical pulses in both time and frequency. We review recent progress in the area and present various applications focussed primarily in the areas of all-optical coding and decoding, and pulse retiming.
\end{abstract}

Keywords: Optical processing, pulse shaping, fibre Bragg gratings, OCDMA

\section{INTRODUCTION}

The capacity and speed requirements of communications systems is set to increase for the foreseeable future and this will ultimately dictate that an increasing amount of the routing and processing of optical signals is carried out in the optical domain. As a consequence there is great interest in the development of new techniques to monitor and control the detailed shape and properties of both individual data pulses and data streams, both in terms of improving the fidelity of the data transmission itself, but also to develop new optical processing capabilities and subsystems. This is becoming ever more important and demanding with the increased interest in new modulation formats which require ever greater control of both the amplitude and phase profiles of the optical signals. In this paper we review progress in the use of superstructured fibre grating technology, which offers a ready route to the production of optical filters with very precisely controlled phase and amplitude profiles and which can therefore be used to manipulate the properties of incident optical signals. A superstructured fibre Bragg grating [1,2], is defined as a standard fibre grating, i.e. a grating with a rapidly varying refractive index modulation of uniform amplitude and pitch, onto which an additional, slowly varying amplitude/phase modulation, has been imposed along its length (as illustrated schematically in Figure 1 for the case of SSFBGs for optical code generation). In the low grating reflectivity limit, i.e. for which the light penetrates the full grating length, then the optical frequency response of the grating is given simply by the Fourier transform of the spatial refractive index modulation profile of the grating. As a result the shape of the impulse response (in the time domain) directly follows the grating refractive index superstructure (in the spatial domain). SSFBG technology thus provides a route to map spatial information directly into the temporal domain. The precision with which the superstructure phase and amplitude profiles can be written using state of the art grating facilities allows for very fine and accurate control of the superstructure profile and thus great control of the SSFBGs spectral phase and amplitude reflectivity. Consequently, if one knows the phase and amplitude of some incident pulse to the grating and one has a target reshaped pulse in mind e.g. a rectangular pulse for example, then one can readily determine the complex spectral reflectivity of the SSFBG required to obtain the necessary pulse reshaping. Given the inherent fibre compatibility, and potential for low cost, this provides a very convenient and powerful way of reshaping pulses in fibre systems.

To illustrate the sort of functionality that these components enable we will focus within this paper on just two applications - the encoding and recognition of optical codes, and the retiming of optical signals (an example of where gratings can be used to enhance the performance of an optical switch based on the use of state-of-the-art, dispersion managed, high nonlinearity fibres). However, it is to be appreciated that such devices have been used to enable a far broader range of applications than this including pulse repetition rate multiplication [3], 2Rregeneration [4], pulse generation and compression [5], data format conversion [6], through to more exotic applications such as Optical Fourier Transformation (OFT) [7]. We hope to have time to touch more fully on these other applications in the associated presentation.

\section{Use of SSFBGs for Optical Code Generation and Recognition}

Optical code/header generation and recognition are required in a number of network concepts including both Optical Code Division Multiple Access (OCDMA) and packet switching. OCDMA is a spread spectrum technique similar to that that has been applied with such great success in wireless communication systems. CDMA techniques allow a large pool of users to share the same transmission bandwidth. Each individual user, or sub-group of users, is allocated a specific address (code) that can be used to label bits that are either to be 
transmitted to the user, or to be transmitted by the user. The optical encoding is ordinarily performed either in the time domain (direct sequence DS-OCDMA), or in the frequency domain (frequency-hopping FH-OCDMA). Hybrid (also called two-dimensional or frequency-hopping) approaches have also been demonstrated. In DSOCDMA each data bit to be transmitted is defined by a code composed of a sequence of individual pulses, referred to as chips. Coded bits are then broadcast onto the network and will only be received by users having a receiver designed to recover data bits encoded with that specific address. A variety of technical approaches to the coding/decoding process have been demonstrated to date including those based on spreading pulses in the time domain using for example arrays of fibre delay lines, planar lightwave circuits, liquid crystal based spatial light modulators, or arrays of fibre gratings. Recently, however superstructured fibre Bragg grating (SSFBG) technology has emerged as an attractive and highly flexible route to produce high performance and potentially low-cost optical coders and decoders for DS-OCDMA.

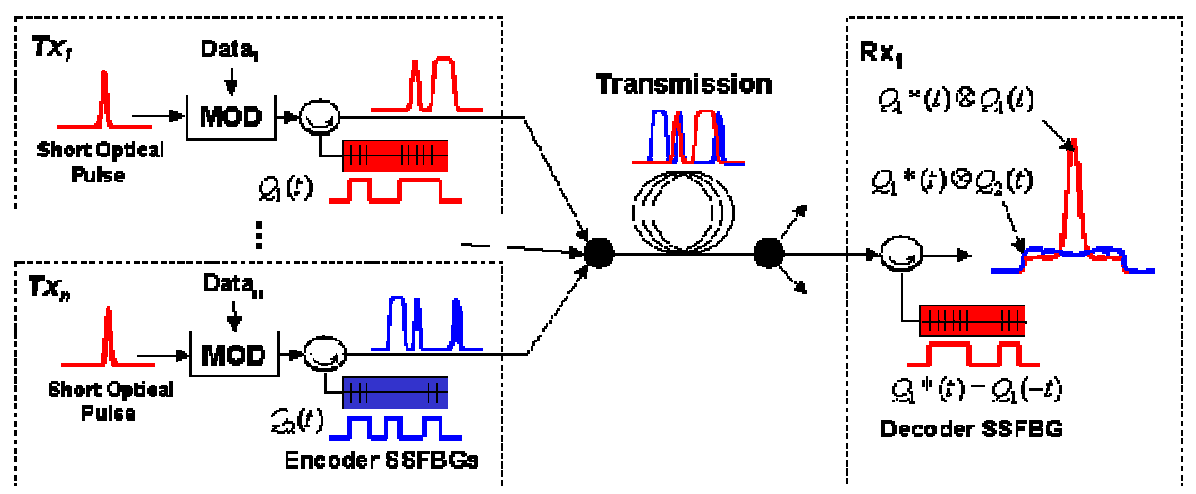

Figure 1 Principle of the SSFBG based code generation and detection

Coded pulses are generated as shown in Figure 1 by reflecting the data from an SSFBG with coding information written into the superstructure profile. This can be either a phase or an amplitude envelope, depending on whether the code is to be phase- or amplitude-shift keyed [8]. Code recognition is obtained by matched filtering the resulting coded signal using a decoder grating with the time reversed (conjugate) impulse response to that of the encoder grating. Such an impulse response is readily obtained using a grating with a spatially reversed superstructure profile relative to that of the encoder grating i.e. by using a grating with exactly the same refractive index profile as the encoder grating and illuminating it from the other end. When the encoder and decoder gratings are correctly matched the matched filtering process results in the generation of a pulse in the time domain that has the same shape as the code's autocorrelation function. In the instance that an 'incorrect' matched filter is used i.e. a code corresponding to another user of the system, the pulse so generated has the shape of the cross correlation of the two codes. Obviously, to get good operation of the system it is important that each user is able to reliably recognize data bits encoded with his particular code, and does not mistakenly receive bits intended for other users. To achieve this it is necessary to restrict the allocation of code sequences only to a subset of all possible code sequences that have both distinct well-defined autocorrelation characteristics, (i.e. a single well defined peak with a width of order the chip duration), and mutually low cross-correlation characteristics. The identification of suitable code sets has received much attention in the context of mobile communications and code sequences such as the M-sequence, Gold and Kasami code sequences can be generated using established mathematical procedures. Such code sequences have been used extensively in the radio communications fields and many of these coding schemes can also be applied to the case of OCDMA systems.

In terms of the technology, SSFBG encoders with both amplitude and up to 4-level phase shift keying have been demonstrated, with code length of up to 511 Chips (at chip rates of up to $640 \mathrm{GChip} / \mathrm{s}$ ) [9]. Current work is now focused more on assessing the system performance of such devices. For example, we have recently demonstrated a 16 user 4 x OCDMA/4 x WDM system with all channels spaced on a 50GHz ITU grid [10]. Note that this system also incorporates a tunable SSFBG decoder comprising an array of fine heating wires along the grating length to induce local thermally induced phase shifts and which allow full code reconfiguration with a $\sim 2$ reconfiguration time [11], and exploits the use of distributed rather than abrupt grating phase shift codes to allow the dense wavelength channel spacings [12]. In Figure 2 we present various plots highlighting the main performance characteristics of this system. As can be seen error free performance was achieved for the case of 16 simultaneous users. It is important to note that the decode SSFBGs simultaneously perform both code recognition and DWDM demultiplexing functions in this system. 

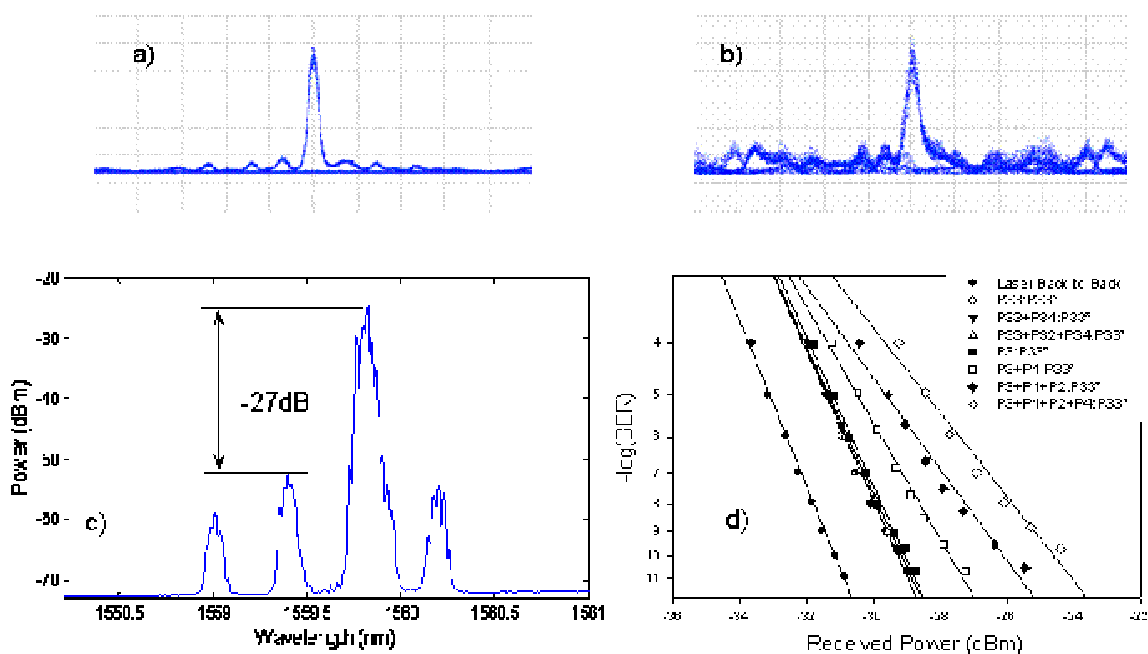

Figure 2 (a) Detection signal for code P3 on wavelength channel 3 in the presence of code p3 on the 3 other wavelength channels (b) Detection signal in the presence of all other 15 channels, (c) Spectrum corresponding to time domain signal (b). (d) BER data for differing numbers of system users showing the impact of multiple access interference noise.

\section{TIMING JITTER REDUCTION}

The reduction of timing jitter is likely to be an essential function within future high speed optical communication systems. Previously, we have demonstrated retiming schemes based on the use of square pulses generated using SSFBGs (in conjunction with NOLM fibre switches [13]), and have now shown capable of operating at data rates as high as 160Gbit/s [14]. Here we demonstrate an alternative approach exploiting parabolic pulses [15]. The generation of parabolic pulses is significant from a nonlinear processing perspective since these pulses generate a strictly linear chirp under the influence of both Self Phase and Cross Phase Modulation (SPM and XPM respectively) offering a large number of device opportunities ranging from pulse compression through to OFT.
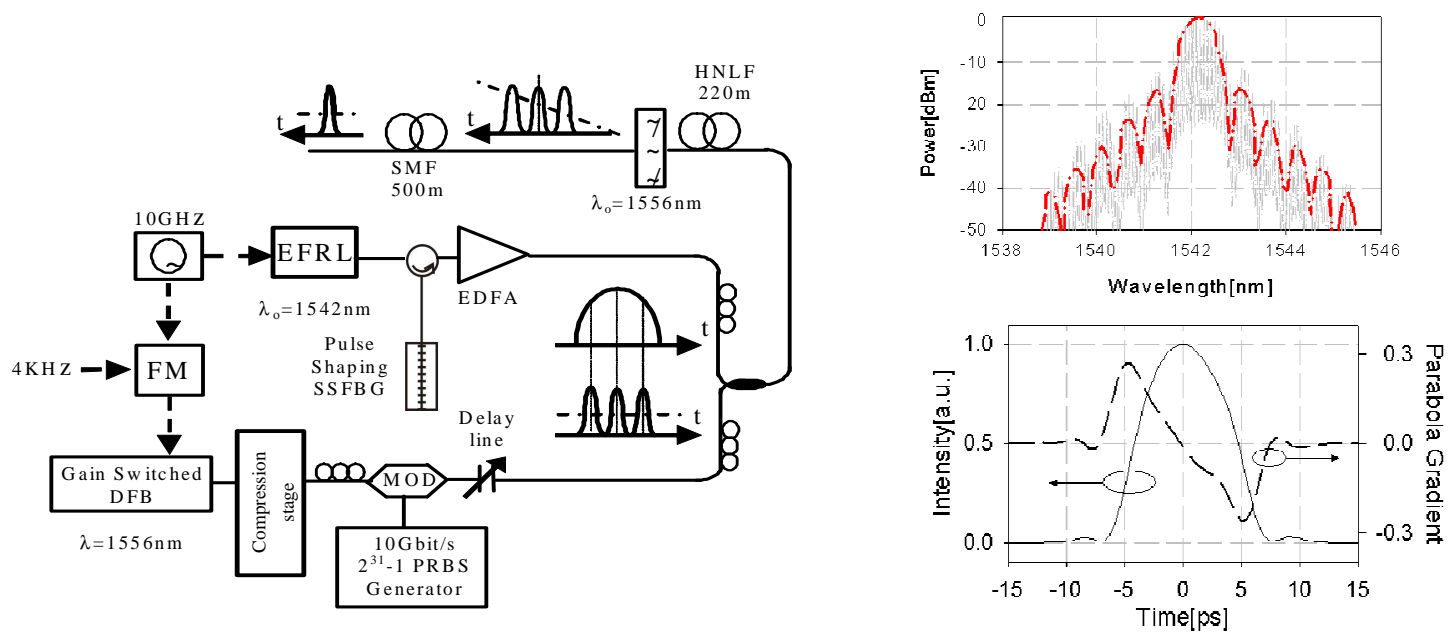

Figure 3 (left) Set up and principle of the parabolic pulse retiming system. (right) (a) Experimental (dotted line) and calculated (dashed-dotted line) spectrum of the parabolic pulses. (b) Intensity of the shaped parabolic pulses measured using SHG-FROG and its corresponding gradient.

The experimental set-up and the retiming scheme principle are shown in Figure 3. The technique is based on the fact that the chirp imparted by an intense parabolic control pulse on a co-propagating signal in a Kerr medium is linear. Thus data pulses offset from the control pulse centre have a frequency offset that is simply proportional to the temporal offset of the data pulses from the control pulse centre. There is thus a one-to-one linear mapping of timing jitter to frequency jitter. By passing the frequency modulated data bits through a suitably dispersive delay line it is possible to retime the data bits on a bit-by bit basis. The key issue with this technique is how to produce the control pulses with a sufficiently accurate parabolic pulse shape. In our experiments we use an SSFBG pulse shaper to generate 10ps parabolic pulses from 2ps incident pulses generated from a mode-locked erbium fibre soliton ring laser (EFRL). The measured optical spectrum of the pulses from the SSFBG is shown in Fig.3b along with the pulse shape as measured using a second harmonic generation frequency-resolved optical gating (SHG-FROG). As can be seen the intensity envelope derivative 
exhibits a reasonably good linear slope across the $10 \mathrm{ps}$ pulsewidth as required. In our experiments the data pulses had a duration of $2 \mathrm{ps}$ and we were able to apply controllable levels of simulated induced timing jitter. We evaluated the quality of our retiming scheme at the system output and these measurements are summarised in Figure 4 , where we also show examples of eye diagrams at the input and output of the retiming scheme, obtained for incoming signals with an RMS timing jitter of $\sim 1.8 \mathrm{ps}$ ((peak-peak (p-p) of $\sim 10.4$ ps), $\sim 2.9$ ps (p-p of $\sim 15.3$ ps) and $\sim 3.5 \mathrm{ps}$ ( $\mathrm{p}-\mathrm{p}$ of $\sim 16.4 \mathrm{ps}$ ) respectively. The measured jitter of the pulses at the output of the retiming scheme cases was measured as $\sim 1$ ps (RMS value) - the resolution limit of the sampling scope used for our measurements. The benefits of the jitter reduction are obvious.

\section{CONCLUSIONS}

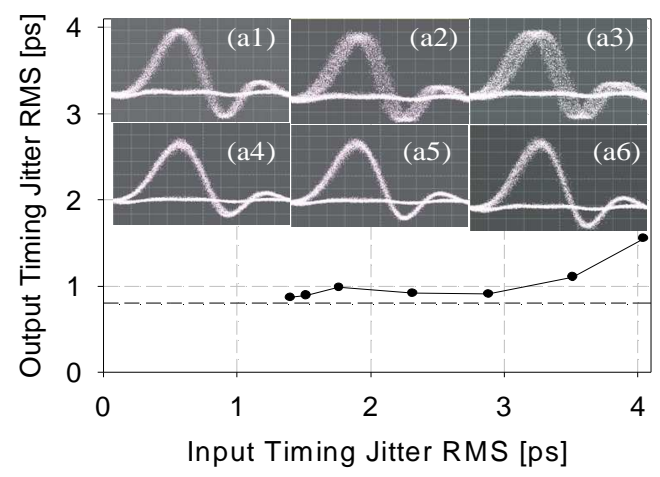

Figure 4 Output versus input RMS timing jitter of the data signal (Inset) Eye diagrams before (a.1-3) and after (a.4-6) the retiming scheme for three different values of induced timing jitter: RMS 1.8ps (p-p 10.4ps), RMS 2.9 (p-p 15.3ps) and RMS 3.5ps (p-p 16.4ps). Time scale: 10ps/div.

In conclusion we have reviewed recent progress in the area of SSFBGs for pulse shaping applications and have shown that reliable, accurate and complex reshaping of picosecond optical pulses can be achieved. We have demonstrated a number of applications of the approach and have referred to others. We consider our results to highlight that this a most promising approach to manipulating and controlling optical signals within future optical networks, and that this technology has many other applications beyond telecommunications also, not least in the area of high power fibre lasers.

\section{REFERENCES}

[1] B.J.Eggleton, P.A.Krug, L.Poladian: Long periodic superstructure Bragg gratings in optical fibers, Electronics letters 1994 Vol 30 (19) pp1620-1622.

[2] M.Ibsen, M.K.Durkin, M.J.Cole, R.I.Laming: Sinc-sampled fibre Bragg gratings for identical multiple wavelength operation, IEEE Photonics Technology Letters 1998 Vol 10 pp 842-4.

[3] P.Petropoulos, M.Ibsen, D.J.Richardson, M.N.Zervas: Generation of a $40 \mathrm{GHz}$ pulse stream by pulse multiplication using a sampled fiber Bragg grating, Optics Letters 2000 Vol.25(8) pp.521-3.

[4] F.Parmigiani, P.Petropoulos, M.Ibsen, D.J.Richardson: All-optical pulse reshaping and retiming systems incorporating pulse shaping fiber Bragg grating, IEEE Journal of Lightwave Technology 2006 Vol.24(1) pp.357-364.

[5] F.Parmigiani, C.Finot, K.Mukasa, M.Ibsen, M.A.F.Roelens, P.Petropoulos, D.J.Richardson: Ultra-flat SPMbroadened spectra in a highly nonlinear fiber using parabolic pulses formed in a fiber Bragg grating, Optics Express 2006 Vol.14(17) pp.7617-7622.

[6] P.J.Almeida, P.Petropoulos, F.Parmigiani, M.Ibsen, D.J.Richardson: OTDM add-drop multiplexer based on timefrequency signal processing, IEEE Journal of Lightwave Technology 2006 Vol.24(7) pp.2720-2732.

[7] T.T.Ng, F.Parmigiani, M.Ibsen, Z.Zhang, P.Petropoulos, D.J.Richardson: Linear-distortion compensation using XPM with parabolic pulses, Proceedings OFC 2007, paper JWA8

[8] P.C.Teh, P.Petropoulos, M.Ibsen, D.J.Richardson: A comparative study of the performance of seven-and 63-chip optical code-division multiple-access encoders and decoders based on superstructured fiber Bragg gratings, IEEE Journal of Lightwave Technology 2001 Vol.19(9) pp.1352-65.

[9] X.Wang , K.Matsushima, K.Kitayama: High-performance optical code generation and recognition by use of a 511chip, 640-Gchip/s phase-shifted superstructured fiber Bragg grating, Optics. Letters 2005 Vol.30 (4) pp. 355-357

[10] C.Tian, Z.Zhang, M.Ibsen, P.Petropoulos, D.J.Richardson: Demonstration of a 16-channel code-reconfigurable OCDMA/DWDM system, Proceedings OFC 2007, paper OMO2.

[11] Z.Zhang, C.Tian, M.R.Mokhtar, P.Petropoulos, D.J.Richardson, M.Ibsen: Rapidly reconfigurable optical phase encoder-decoders based on fiber Bragg gratings, IEEE Photonics Technology Letters 2006 Vol.18(11) pp.1216-1218

[12] Z.Zhang, C.Tian, P.Petropoulos, D.J.Richardson, M.Ibsen: Fibre Bragg grating based continuous-phase encoderdecoders for OCDMA networks, Proceedings ECOC 2006 , paper We3.P.38

[13] J H.Lee, P.C.Teh, P.Petropoulos, M.Ibsen, D.J.Richardson: All optical modulation and demultiplexing systems with significant timing jitter tolerance through incorporation of pulse-shaping fiber Bragg gratings, IEEE Photonics Technology Letters 2002 Vol.14(2) pp.203-5.

[14] L.K.Oxenlowe, F.Parmigiani, M.Galili, D.Zibar, A.T.Clausen, M.Ibsen, P.Petropoulos, D.J.Richardson, P.Jeppeson: $160 \mathrm{~Gb} / \mathrm{s}$ retiming using rectangular pulses generated using a superstructured fibre Bragg grating, Proceedings OECC/IOOC 2007 paper 13B3-4

[15] F.Parmigiani, P.Petropoulos, M.Ibsen, D.J.Richardson: Pulse retiming based on XPM using parabolic pulses formed in a fiber Bragg grating, IEEE Photonics Technology Letters 2006 Vol.18(7) pp.829-831. 